\title{
NeW RESUlts ON THE OUTPUT REgulation OF THE MODIFIED ARNEODO CHAOTIC SYSTEM BY STATE FEEDBACK CONTROL
}

\author{
Sundarapandian Vaidyanathan ${ }^{1}$ \\ ${ }^{1}$ Research and Development Centre, Vel Tech Dr. RR \& Dr. SR Technical University \\ Avadi, Chennai-600 062, Tamil Nadu, INDIA \\ sundarvtulgmail.com
}

\begin{abstract}
This paper investigates the problem of output regulation of the modified Arneodo chaotic system, which is one of the classical chaotic systems proposed by A. Arneodo, P. Coullet and C. Tresser (1981). Explicitly, new state feedback control laws regulating the output of the modified Arneodo chaotic system have been derived so as to regulate the output of the modified Arneodo chaotic system have been derived so as to track the constant reference signals as well as periodic reference signals. The control laws proposed in this paper have been derived using the regulator equations of C.I. Byrnes and A. Isidori (1990), who solved the problem of output regulation of nonlinear control systems involving neutrally stable exosystem dynamics. The output regulation of the modified Arneodo chaotic system has important applications in Electronics and Communication Engineering. Numerical simulations are shown to illustrate the effectiveness of the control schemes proposed in this paper for the output regulation of the modified Arneodo chaotic system.
\end{abstract}

\section{KEYWORDS}

Chaos; feedback control; modified Arneodo system; nonlinear control systems; output regulation.

\section{INTRODUCTION}

Regulating the output of control systems is one of the core problems in control systems theory. Basically, the problem of output regulation is to control a fixed linear or nonlinear plant in order to have its output tracking reference signals produced by some external generator (the exosystem). For linear control systems, the output regulation problem has been solved by Francis and Wonham ([1], 1975). For nonlinear control systems, the output regulation problem has been solved by Byrnes and Isidori ([2], 1990) generalizing the internal model principle obtained by Francis and Wonham [1]. Using Centre Manifold Theory [3], Byrnes and Isidori have derived regulator equations, which completely characterize the solution of the output regulation problem of nonlinear control systems.

The output regulation problem for nonlinear control systems has been studied extensively by various scholars in the last two decades [4-14]. In [4], Mahmoud and Khalil obtained results on the asymptotic regulation of minimum phase nonlinear systems using output feedback. In [5], Fridman solved the output regulation problem for nonlinear control systems with delay using centre manifold theory. In [6-7], Chen and Huang obtained results on the robust output regulation for output feedback systems with nonlinear exosystems. In [8], Liu and Huang obtained results on the global robust output regulation problem for lower triangular nonlinear systems with unknown control direction. In [9], Immonen obtained results on the practical output regulation for bounded linear infinite-dimensional state space systems. In [10], Pavlov, Van de Wouw and Nijmeijer obtained results on the global nonlinear output regulation using convergence-based controller design. In [11], $\mathrm{Xi}$ and Dong obtained results on the global adaptive output regulation of a class of nonlinear systems with nonlinear exosystems. In [12-

DOI : 10.5121/acij.2011.2202 
14], Serrani, Isidori and Marconi obtained results on the semi-global and global output regulation problem for minimum-phase nonlinear systems.

In this paper, we solve the output regulation problem for the modified Arneodo chaotic system ([15], 1981). We derive state feedback control laws solving the output regulation problem of the modified Arneodo chaotic system using the regulator equations of Byrnes and Isidori (1990) for the important cases of constant and periodic reference signals. The modified Arneodo system is a classical three-dimensional chaotic system studied by A. Arneodo, P. Coullet and C. Tresser (1981). It has important applications in Electronics and Communication Engineering.

This paper is organized as follows. In Section 2, we provide a review the problem statement of output regulation problem for nonlinear control systems and the regulator equations of Byrnes and Isidori [2], which provide a solution to the output regulation problem under some stability assumptions. In Section 3, we present the main results of this paper, namely, the solution of the output regulation problem for the modified Arneodo chaotic system for the important cases of constant and periodic reference signals. In Section 4, we describe the numerical results illustrating the effectiveness of the control schemes derived in Section 3 for the solution of the output regulation problem for the modified Arneodo chaotic system. Section 5 summarizes the main results obtained in this paper.

\section{Review of the Output Regulation Problem for Nonlinear CONTROL SYSTEMS}

In this section, we consider a multi-variable nonlinear control system described by

$$
\begin{aligned}
& \dot{x}=f(x)+g(x) u+p(x) \omega \\
& \dot{\omega}=s(\omega) \\
& e=h(x)-q(\omega)
\end{aligned}
$$

Here, the differential equation (1a) describes the plant dynamics with state $x$ defined in a neighbourhood $X$ of the origin of $R^{n}$ and the input $u$ takes values in $R^{m}$ subject to te effect of a disturbance represented by the vector field $p(x) \omega$. The differential equation (1b) describes an autonomous system, known as the exosystem, defined in a neighbourhood $W$ of the origin of $R^{k}$, which models the class of disturbance and reference signals taken into consideration. The equation (2) defines the error between the actual plant output $h(x) \in R^{p}$ and a reference signal $q(\omega)$, which models the class of disturbance and reference signals taken into consideration.

We also assume that all the constituent mappings o the system (1) and the error equation (2), namely, $f, g, p, s, h$ and $q$ are continuously differentiable mappings vanishing at the origin.

Thus, for $u=0$, the composite system (1) has an equilibrium $(x, \omega)=(0,0)$ with zero error (2).

A state feedback controller for the composite system (1) has the form

$$
u=\rho(x, \omega)
$$

where $\rho$ is a continuously differentiable mapping defined on $X \times W$ such that $\rho(0,0)=0$. 
Upon substitution of the feedback control law (3) into (1), we get the closed-loop system

$$
\begin{aligned}
& \dot{x}=f(x)+g(x) \rho(x, \omega)+p(x) \omega \\
& \dot{\omega}=s(\omega)
\end{aligned}
$$

The purpose of designing the state feedback controller (3) is to achieve both internal stability and output regulation of the given nonlinear control system (1). Formally, we can summarize these requirements as follows.

\section{State Feedback Regulator Problem [2]:}

Find, if possible, a state feedback control law $u=\rho(x, \omega)$ such that the following conditions are satisfied.

(OR1) [Internal Stability] The equilibrium $x=0$ of the dynamics

$$
\dot{x}=f(x)+g(x) \rho(x, 0)
$$

is locally exponentially stable.

(OR2) [Output Regulation] There exists a neighbourhood $U \subset X \times W$ of $(x, \omega)=(0,0)$ such that for each initial condition $(x(0), \omega(0)) \in U$, the solution $(x(t), \omega(t))$ of the closed-loop system (4) satisfies

$$
\lim _{t \rightarrow \infty}[h(x(t))-q(\omega(t))]=0 .
$$

Byrnes and Isidori [2] solved the output regulation problem stated above under the following two assumptions.

(H1) The exosystem dynamics $\dot{\omega}=s(\omega)$ is neutrally stable at $\omega=0$, i.e. the exosystem is Lyapunov stable in both forward and backward time at $\omega=0$.

(H2) The pair $(f(x), g(x))$ has a stabilizable linear approximation at $x=0$, i.e. if

$$
A=\left[\frac{\partial f}{\partial x}\right]_{x=0} \text { and } B=\left[\frac{\partial g}{\partial x}\right]_{x=0},
$$

then $(A, B)$ is stabilizable.

Next, we recall the solution of the output regulation problem derived by Byrnes and Isidori [2].

Theorem 1. [2] Under the hypotheses (H1) and (H2), the state feedback regulator problem is solvable if and only if there exist continuously differentiable mappings $x=\pi(\omega)$ with $\pi(0)=0$ and $u=\varphi(\omega)$ with $\varphi(0)=0$, both defined in a neighbourhood of $W^{0} \subset W$ of $\omega=0$ such that the following equations (called the regulator equations) are satisfied: 
Advanced Computing: An International Journal ( ACIJ ), Vol.2, No.2, March 2011

(1) $\frac{\partial \pi}{\partial \omega} s(\omega)=f(\pi(\omega))+g(\pi(\omega)) \varphi(\omega)+p(\pi(\omega)) \omega$

(2) $h(\pi(\omega))-q(\omega)=0$

When the regulator equations (1) and (2) are satisfied, a control law solving the state feedback regulator problem is given by

$$
u=\varphi(\omega)+K[x-\pi(\omega)]
$$

where $K$ is any gain matrix such that $A+B K$ is Hurwitz.

\section{Output Regulation of the Modified Arneodo Chaotic System}

In this section, we solve the output regulation problem for the modified Arneodo chaotic system. The modified Arneodo chaotic system ([15], 1981) is one of the paradigms of the threedimensional chaotic systems described by the dynamics

$$
\begin{aligned}
& \dot{x}_{1}=x_{2} \\
& \dot{x}_{2}=x_{3} \\
& \dot{x}_{3}=\alpha x_{1}-\beta x_{2}-x_{3}-x_{1}^{3}+u
\end{aligned}
$$

where $\alpha>0, \beta>0$ are parameters of the system (5) and $u$ is the control.

A. Arneodo, P. Coullet and C. Tresser [15] showed that the system (5) has chaotic behaviour when $\alpha=5, \beta=3.8$ and $u=0$. The chaotic portrait of the modified Arneodo chaotic system (5) is illustrated in Figure 1.

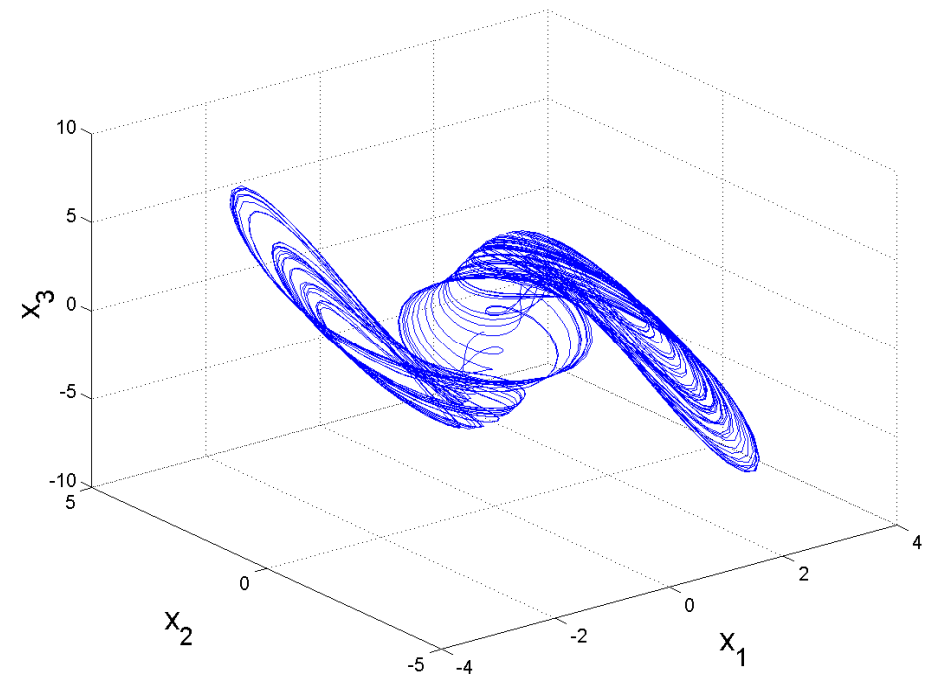

Figure 1. Chaotic Portrait of the Modified Arneodo System 
Advanced Computing: An International Journal ( ACIJ ), Vol.2, No.2, March 2011

In this paper, we consider the output regulation problem for two important cases, viz.

(I) Tracking of Constant Reference Signals

(II) Tracking of Periodic Reference Signals

\section{Case I: Tracking of Constant Reference Signals}

In this case, the exosystem is given by the scalar dynamics

$$
\dot{\omega}=0
$$

We note that the assumption (H1) of Theorem 1 holds trivially.

Linearizing the dynamics of the modified Arneodo system (5) at $(x, \omega)=(0,0)$, we obtain

$$
A=\left[\begin{array}{ccc}
0 & 1 & 0 \\
0 & 0 & 1 \\
\alpha & -\beta & -1
\end{array}\right] \quad \text { and } B=\left[\begin{array}{l}
0 \\
0 \\
1
\end{array}\right]
$$

Using Kalman's rank test for controllability ([16], p738), it can be easily seen that the pair $(A, B)$ is completely controllable. Since $(A, B)$ is in Bush companion form, the characteristic equation of $A+B K$ is given by

$$
\lambda^{3}+\left(1-k_{3}\right) \lambda^{2}+\left(\beta-k_{2}\right) \lambda-\left(\alpha+k_{1}\right)=0
$$

where $K=\left[\begin{array}{lll}k_{1} & k_{2} & k_{3}\end{array}\right]$.

By the Routh's stability criterion ([16], p.234), it can be easily shown that the closed-loop system matrix $A+B K$ is Hurwitz if and only if

$$
k_{1}<-\alpha, k_{2}<\beta, k_{3}<1,\left(1-k_{3}\right)\left(\beta-k_{2}\right)+\left(\alpha+k_{1}\right)>0
$$

Thus, the assumption (H2) of Theorem 1 also holds. Hence, Theorem 1 can be applied to solve the output regulation problem for the modified Arneodo chaotic system (5) for the tracking of constant reference signals (set-point signals).

\section{Case I (a): The constant tracking problem for $x_{1}$}

Here, the tracking problem for the modified Arneodo chaotic system is given by

$$
\begin{aligned}
& \dot{x}_{1}=x_{2} \\
& \dot{x}_{2}=x_{3} \\
& \dot{x}_{3}=\alpha x_{1}-\beta x_{2}-x_{3}-x_{1}^{3}+u \\
& e=x_{1}-\omega
\end{aligned}
$$


Advanced Computing: An International Journal ( ACIJ ), Vol.2, No.2, March 2011

Solving the regulator equations for the system (9), we obtain the unique solution as

$$
\pi_{1}(\omega)=\omega, \quad \pi_{2}(\omega)=0, \quad \pi_{3}(\omega)=0 \text { and } \varphi(\omega)=-\alpha \omega+\omega^{3} .
$$

Using Theorem 1 and the solution (10) of the regulator equations for the system (9), we obtain the following result which provides a solution of the output regulation problem.

Theorem 2. A state feedback control law solving the output regulation problem for the modified Arneodo chaotic system (9) is given by

$$
u=-\alpha \omega+\omega^{3}+k_{1}\left(x_{1}-\omega\right)+k_{2} x_{2}+k_{3} x_{3},
$$

where $k_{1}, k_{2}$ and $k_{3}$ satisfy the inequalities (8).

\section{Case I (b): The constant tracking problem for $x_{2}$}

Here, the tracking problem for the modified Arneodo chaotic system is given by

$$
\begin{aligned}
& \dot{x}_{1}=x_{2} \\
& \dot{x}_{2}=x_{3} \\
& \dot{x}_{3}=\alpha x_{1}-\beta x_{2}-x_{3}-x_{1}^{3}+u \\
& e=x_{2}-\omega
\end{aligned}
$$

It is easy to show that the regulator equations for the system (12) are not solvable. Thus, by Theorem 1, the output regulation problem is not solvable for this case.

\section{Case I (c): The constant tracking problem for $x_{3}$}

Here, the tracking problem for the modified Arneodo chaotic system is given by

$$
\begin{aligned}
& \dot{x}_{1}=x_{2} \\
& \dot{x}_{2}=x_{3} \\
& \dot{x}_{3}=\alpha x_{1}-\beta x_{2}-x_{3}-x_{1}^{3}+u \\
& e=x_{3}-\omega
\end{aligned}
$$

It is easy to show that the regulator equations for the system (13) are not solvable. Thus, by Theorem 1, the output regulation problem is not solvable for this case.

\section{Case II: Tracking of Periodic Reference Signals}

In this case, the exosystem is given by the two-dimensional dynamics

$$
\begin{aligned}
& \dot{\omega}_{1}=v \omega_{2} \\
& \dot{\omega}_{2}=-v \omega_{1}
\end{aligned}
$$

where $v>0$ is a constant. 
It is important to observe that the exosystem given by (14) is neutrally stable. Thus, the assumption (H1) of Theorem 1 holds. Also, as stated earlier, the system linearization pair $(A, B)$ of the modified Arneodo system is completely controllable. We assume that the gain matrix $K$ satisfies the inequalities (8) so that $A+B K$ is Hurwitz.

Hence, Theorem 1 can be applied to solve the output regulation problem for the modified Arneodo chaotic system (5) for the tracking of periodic reference signals generated by the exosystem dynamics (14).

\section{Case II (a): Periodic Tracking Problem for $x_{1}$}

Here, the tracking problem for the modified Arneodo system is given by

$$
\begin{aligned}
& \dot{x}_{1}=x_{2} \\
& \dot{x}_{2}=x_{3} \\
& \dot{x}_{3}=\alpha x_{1}-\beta x_{2}-x_{3}-x_{1}^{3}+u \\
& e=x_{1}-\omega_{1}
\end{aligned}
$$

Solving the regulator equations for the system (15), we obtain the unique solution as

$$
\begin{aligned}
& \pi_{1}(\omega)=\omega_{1} \\
& \pi_{2}(\omega)=v \omega_{2} \\
& \pi_{3}(\omega)=-v^{2} \omega_{1} \\
& \varphi(\omega)=-\left(\alpha+v^{2}\right) \omega_{1}+\omega_{1}^{3}+v \omega_{2}\left(\beta-v^{2}\right)
\end{aligned}
$$

Using Theorem 1 and the solution (16) of the regulator equations for the system (15), we obtain the following result which provides a solution to the output regulation problem.

Theorem 3. A state feedback control law solving the output regulation problem for the system (15) is given by

$$
u=\varphi(\omega)+k_{1}\left(x_{1}-\omega_{1}\right)+k_{2}\left(x_{2}-v \omega_{2}\right)+k_{3}\left(x_{3}+v^{2} \omega_{1}\right)
$$

where $\varphi(\omega)$ is given by (16) and $k_{1}, k_{2}, k_{3}$ satisfy the inequalities (8).

\section{Case II (b): Periodic Tracking Problem for $x_{2}$}

Here, the tracking problem for the modified Arneodo system is given by

$$
\begin{aligned}
& \dot{x}_{1}=x_{2} \\
& \dot{x}_{2}=x_{3} \\
& \dot{x}_{3}=\alpha x_{1}-\beta x_{2}-x_{3}-x_{1}^{3}+u \\
& e=x_{2}-\omega_{1}
\end{aligned}
$$


Solving the regulator equations for the system (18), we obtain the unique solution as

$$
\begin{aligned}
& \pi_{1}(\omega)=-v^{-1} \omega_{2} \\
& \pi_{2}(\omega)=\omega_{1} \\
& \pi_{3}(\omega)=v \omega_{2} \\
& \varphi(\omega)=\left(\beta-v^{2}\right) \omega_{1}+\left(v+\alpha v^{-1}\right) \omega_{2}-v^{-3} \omega_{2}^{3}
\end{aligned}
$$

Using Theorem 1 and the solution (19) of the regulator equations for the system (18), we obtain the following result which gives a solution to the output regulation problem.

Theorem 4. A state feedback control law solving the output regulation problem for the system (18) is given by

$$
u=\varphi(\omega)+k_{1}\left(x_{1}+v^{-1} \omega_{2}\right)+k_{2}\left(x_{2}-\omega_{1}\right)+k_{3}\left(x_{3}-v \omega_{2}\right)
$$

where $\varphi(\omega)$ is given by (19) and $k_{1}, k_{2}, k_{3}$ satisfy the inequalities (8).

\section{Case II (c): Periodic Tracking Problem for $x_{3}$}

Here, the tracking problem for the modified Arneodo system is given by

$$
\begin{aligned}
& \dot{x}_{1}=x_{2} \\
& \dot{x}_{2}=x_{3} \\
& \dot{x}_{3}=\alpha x_{1}-\beta x_{2}-x_{3}-x_{1}^{3}+u \\
& e=x_{3}-\omega_{1}
\end{aligned}
$$

Solving the regulator equations for the system (21), we obtain the unique solution as

$$
\begin{aligned}
& \pi_{1}(\omega)=-v^{-2} \omega_{1} \\
& \pi_{2}(\omega)=-v^{-1} \omega_{2} \\
& \pi_{3}(\omega)=\omega_{1} \\
& \varphi(\omega)=\left(1+\alpha v^{-2}\right) \omega_{1}+\left(v-\beta v^{-1}\right) \omega_{2}-v^{-6} \omega_{1}^{3}
\end{aligned}
$$

Using Theorem 1 and the solution (22) of the regulator equations for the system (21), we obtain the following result which gives a state feedback control law solving the output regulation problem for the system (21).

Theorem 5. A state feedback control law solving the output regulation problem for the system (21) is given by

$$
u=\varphi(\omega)+k_{1}\left(x_{1}+v^{-2} \omega_{1}\right)+k_{2}\left(x_{2}+v^{-1} \omega_{2}\right)+k_{3}\left(x_{3}-\omega_{1}\right)
$$

where $\varphi(\omega)$ is given by (22) and $k_{1}, k_{2}, k_{3}$ satisfy the inequalities (8). 
Advanced Computing: An International Journal ( ACIJ ), Vol.2, No.2, March 2011

\section{NUMERICAL SimUlationS}

For simulation, the parameters are chosen as the chaotic case of the modified Arneodo system, viz. $\alpha=5$ and $\beta=3.8$.

For achieving internal stability of the state feedback regulator problem, a feedback gain matrix $K$ must be chosen so that $A+B K$ is Hurwitz. We choose $K$ so that $A+B K$ has the eigenvalues $\{-4,-4,-4\}$. A simple calculation yields $K=\left[\begin{array}{lll}-69 & -44.2 & -11\end{array}\right]$.

For the periodic tracking output regulation problem, we take the value $v=1$ in the exosystem dynamics (14).

For the numerical simulations, the fourth order Runge-Kutta method with step-size $h=10^{-6}$ is deployed to solve the systems of differential equations using MATLAB.

\section{Tracking of Constant Reference Signals}

\section{I (a) Constant Tracking Problem for $x_{1}$}

Here, the initial conditions are taken as

$$
x_{1}(0)=20, x_{2}(0)=8, x_{3}(0)=16 \text { and } \omega=2 .
$$

The simulation graph is depicted in Figure 2 from which it is clear that the state trajectory $x_{1}(t)$ tracks the constant reference signal $\omega=2$ in 2.8 seconds.

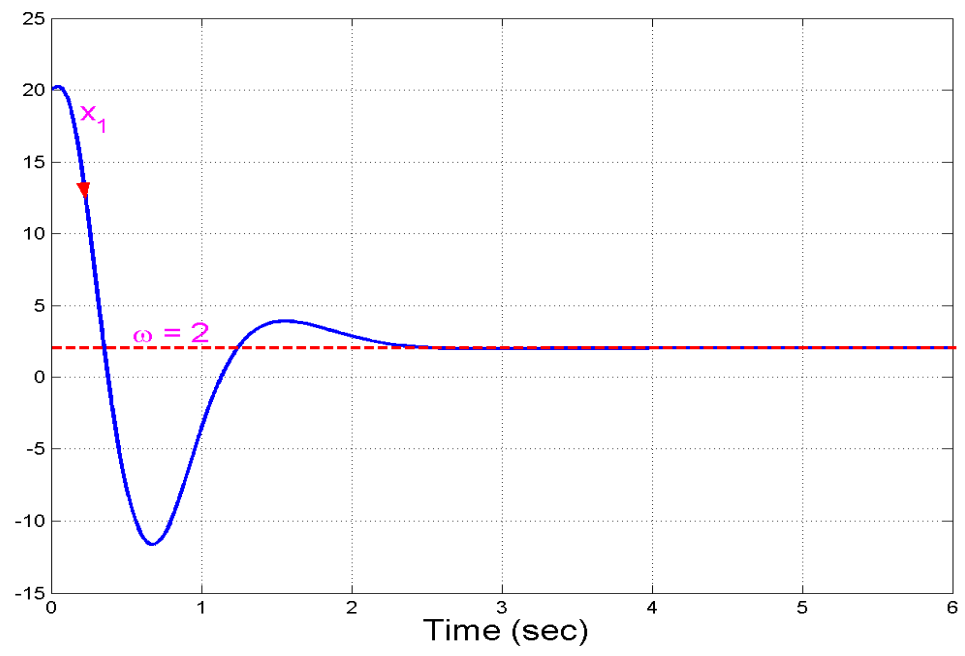

Figure 2. Constant Tracking Problem for $x_{1}$

\section{I (b) Constant Tracking Problem for $x_{2}$}

As pointed out in Section 3, the output regulation problem is not solvable for this case because the regulator equations (14) do not admit any solution. 
Advanced Computing: An International Journal ( ACIJ ), Vol.2, No.2, March 2011

\section{I (c) Constant Tracking Problem for $x_{3}$}

As pointed out in Section 3, the output regulation problem is not solvable for this case because the regulator equations (16) do not admit any solution.

\section{Tracking of Periodic Reference Signals}

Here, it is assumed that $v=1$.

\section{II (a) Periodic Tracking Problem for $x_{1}$}

Here, the initial conditions are taken as

$$
x_{1}(0)=10, x_{2}(0)=20, x_{3}(0)=15, \omega_{1}(0)=1, \omega_{2}(0)=0 .
$$

The simulation graph for this case is depicted in Figure 3 from which it is clear that the state trajectory $x_{1}(t)$ tracks the periodic reference signal $\omega_{1}(t)=\cos t$ in 3 seconds.

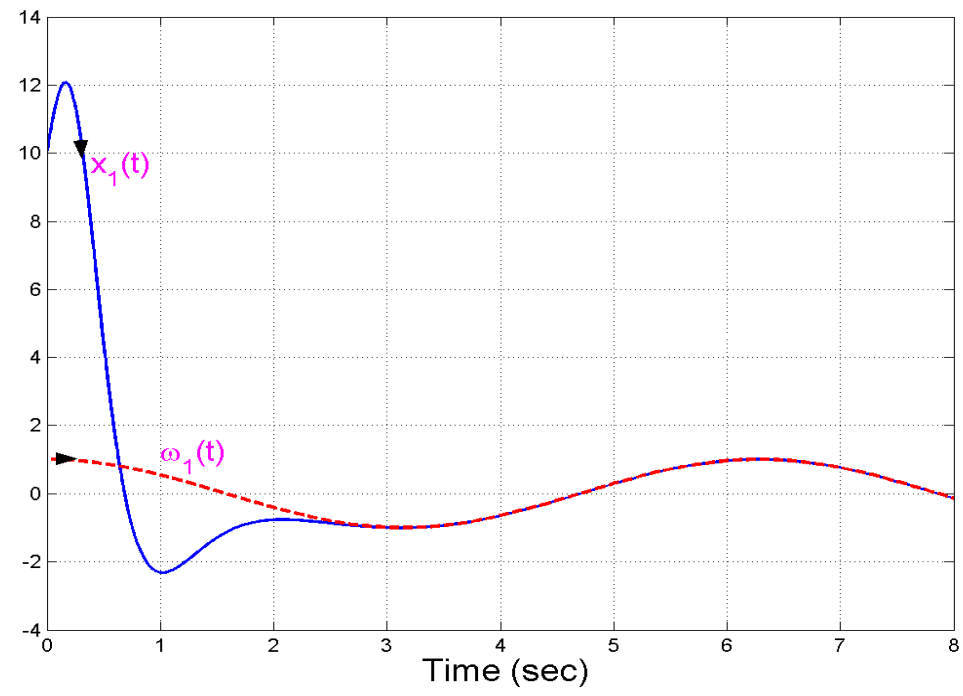

Figure 3. Periodic Tracking Problem for $x_{1}$

\section{II (b) Periodic Tracking Problem for $x_{2}$}

Here, the initial conditions are taken as

$$
x_{1}(0)=6, x_{2}(0)=4, x_{3}(0)=3, \omega_{1}(0)=1, \omega_{2}(0)=0 .
$$

The simulation graph for this case is depicted in Figure 4 from which it is clear that the state trajectory $x_{2}(t)$ tracks the periodic reference signal $\omega_{1}(t)=\cos t$ in 3 seconds. 
Advanced Computing: An International Journal ( ACIJ ), Vol.2, No.2, March 2011

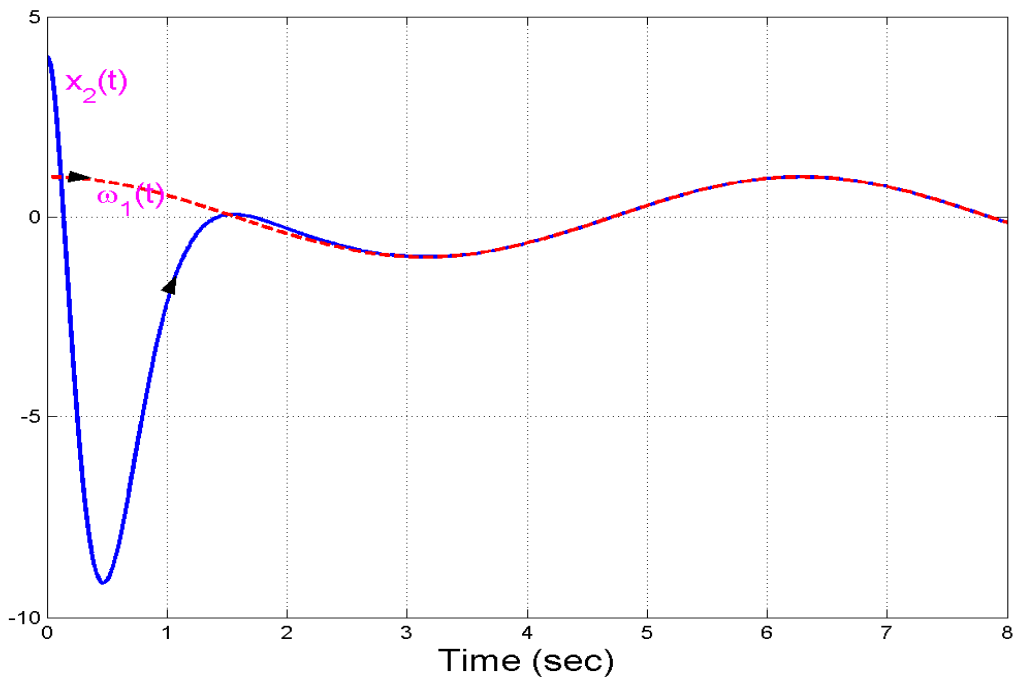

Figure 4. Periodic Tracking Problem for $x_{2}$

\section{II (c) Periodic Tracking Problem for $x_{3}$}

Here, the initial conditions are taken as

$$
x_{1}(0)=3, x_{2}(0)=2, x_{3}(0)=5, \omega_{1}(0)=1, \omega_{2}(0)=0 .
$$

The simulation graph for this case is depicted in Figure 5 from which it is clear that the state trajectory $x_{3}(t)$ tracks the periodic reference signal $\omega_{1}(t)=\cos t$ in 3 seconds.

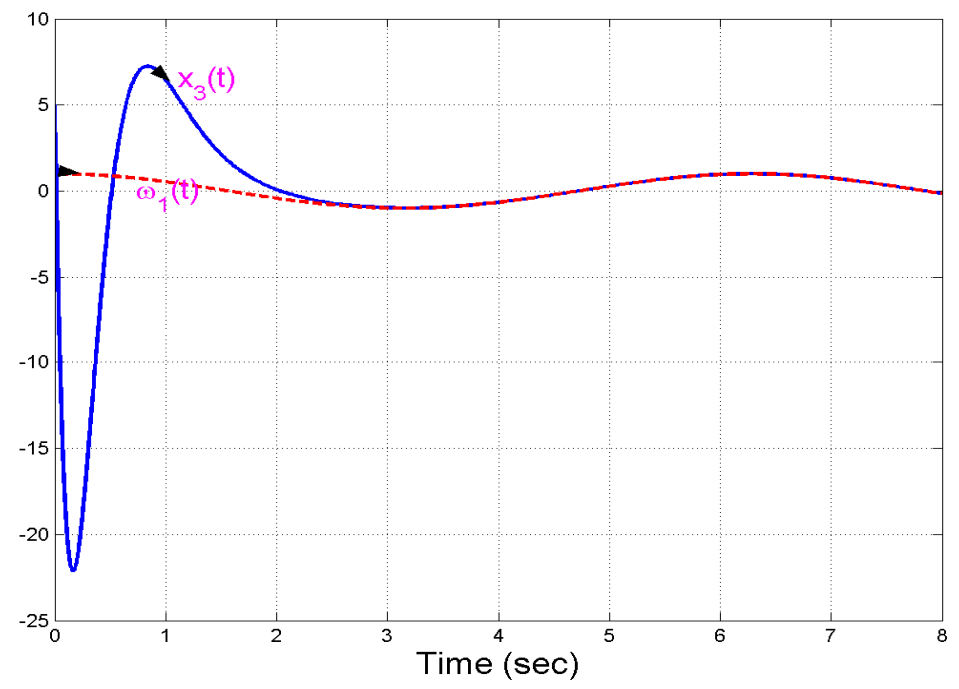

Figure 5. Periodic Tracking Problem for $x_{3}$ 


\section{Conclusions}

In this paper, the output regulation problem for the modified Arneodo chaotic system (1981) has been investigated in detail and a complete solution for the output regulation problem for the modified Arneodo system has been derived for the tracking of constant and periodic reference signals. The state feedback control laws achieving output regulation proposed in this paper were derived using the regulator equations of Byrnes and Isidori (1990). Numerical simulation results were presented in detail to illustrate the effectiveness of the proposed control schemes for the output regulation problem of modified Arneodo system to track constant as well as periodic reference signals.

\section{REFERENCES}

[1] Francis, B.A. \& Wonham, W.M. (1975) "The internal model principle for linear multivariable regulators", J. Applied Math. Optimization, Vol. 2, pp 170-194.

[2] Byrnes, C.I. \& Isidori, A. (1990) "Output regulation of nonlinear systems", IEEE Trans. Automatic Control, Vol. 35, pp 131-140.

[3] Carr, J. (1981) Applications of Centre Manifold Theory, Springer Verlag, New York.

[4] Mahmoud, N.A. \& Khalil, H.K. (1996) "Asymptotic regulation of minimum phase nonlinear systems using output feedback”, IEEE Trans. Automat. Control, Vol. 41, pp 1402-1412.

[5] Fridman, E. (2003) "Output regulation of nonlinear control systems with delay", Systems \& Control Lett., Vol. 50, pp 81-93.

[6] Chen, Z. \& Huang, J. (2005) "Robust output regulation with nonlinear exosystems", Automatica, Vol. 41, pp 1447-1454.

[7] Chen, Z. \& Huang, J. (2005) "Global robust output regulation for output feedback systems", IEEE Trans. Automat. Control, Vol. 50, pp 117-121.

[8] Liu, L. \& Huang, J. (2008) "Global robust output regulation of lower triangular systems with unknown control direction”, Automatica, Vol. 44, pp. 1278-1284.

[9] Immonen, E. (2007) "Practical output regulation for bounded linear infinite-dimensional state space systems", Automatica, Vol. 43, pp 786-794.

[10] Pavlov, A., Van de Wouw, N. \& Nijmeijer, H. (2007) "Global nonlinear output regulation: convergence based controller design”, Automatica, Vol. 43, pp 456-463.

[11] Xi, Z. \& Ding, Z. (2007) "Global robust output regulation of a class of nonlinear systems with nonlinear exosystems”, Automatica, Vol. 43, pp 143-149.

[12] Serrani, A. \& Isidori, A. (2000) "Global robust output regulation for a class of nonlinear systems”, Systems \& Control Lett., Vol. 39, pp 133-139.

[13] Serrani, A., Isidori, A. \& Marconi, L. (2000) "Semiglobal output regulation for minimum phase systems", Internat. J. Robust Nonlinear Control, Vol. 10, pp 379-396.

[14] Marconi, L., Isidori, A. \& Serrani, A. (2004) "Non-resonance conditions for uniform observability in the problem of nonlinear output regulation", Systems \& Control Letters, Vol. 53, pp 281-298.

[15] Arneodo, A., Coullet, P. \& Tresser, C. (1981) "Possible new strange attractors with spiral structure", Commun. Math. Physics, Vol. 79, pp 573-579.

[16] Ogata, K. (1997) Modern Control Engineering, Prentice Hall, New Jersey, U.S.A.

[17] Kamaraju, M., Kishore, K.L. \& Tilak, A.V.N. (2010) "Power optimized programmable embedded controller", Internat. Journal of Computer Networks \& Commun., Vol. 2, pp. 97-107. 